\title{
Launching Of Immunization with the Vaccine Mycobacterium Indicus Pranii for Eradication of Leprosy in India
}

\author{
G.P. Talwar* and Jagdish C. Gupta
}

Talwar Research Foundation, New Delhi, India

Received: 16 June, 2017; Accepted: 26 June, 2017; Published: 29 September, 2017

*Corresponding author: G P Talwar, Director Research, Talwar research, Foundation, E-8, Neb Valley, Neb Sarai, New Delhi-110068, India, Tel: 91-011-65022405; E-mail : gptalwar@gmail.com

\begin{abstract}
Vaccines are developed to mobilize the immune system of the recipient to react against an invading micro-organism and thus prevent infection to occur in the body. Besides immuno-prophylaxis, several vaccines have also revealed their precious immuno-therapeutic properties. Yet another highly important function of vaccines is their utilization for "Eradication" of a disease. Eradication of small pox could not have been achieved without a vaccine. The same is being achieved for elimination of Poliomyelitis in several countries with the massive immunization of the children with the available vaccines, oral and/or injectable. MIP is launched for eradication of leprosy.
\end{abstract}

Keywords: Tuberculosis; Ano-genital warts ; Myeloma ; Adjuvant

\section{Introduction}

This article reports a historically important step that the Ministry of Health, Govt of India, has taken on May 7, 2017, to launch an immunization project to eradicate leprosy from India.

Leprosy is not native to India. The causative micro-organism Mycobacterium leprae was in fact discovered by Armauer Hansen in Norway two centuries back. Although armadillos can host $M$. leprae, humans appear to be the main reservoir of infection. $M$. leprae grows in multibacillary leprosy patients, who shed the bacteria to infect others around them. Luckily 99\% of humans are resistant to invading $M$. leprae (because of their competent immune system). The few who fall victim, sustain M. leprae in the community.

India is one of the few countries of the world, where leprosy is still prevalent. In fact in 2015, a total of 127,326 new cases were identified which account for $60 \%$ of the global total of new cases (www.who.int/lep/en). The Govt of India has been employing a multi-drugs regime to treat free of charge leprosy patients. This has brought down the prevalence rate significantly over the years.

However since 2006, the prevalence rate is static despite intensive programme of treatment with drugs. Moreover the incidence of new cases is on increase (Figure. 1).
An expert committee of the Indian Council of Medical Research along with the Directorate of Health Services has decided to employ the vaccine based on autoclaved Mycobacterium indicus pranii (MIP) in addition to Drugs for treatment of leprosy patients. Their family members and immediate contacts will also be immunized with 2 doses of the vaccine at 6 months interval. The Project will initially be launched in 5 districts of high endemicity and extended to other districts on basis of the observed efficacy of immunization with this vaccine.

\section{Mycobacterium Indicus Pranii (MIP)}

MIP vaccine was developed by me along with my co-workers to overcome the inability of leprosy patients to react to some key antigens of M. leprae. Extensive studies were carried out to understand the nature of immune deficit in those who contract the disease on exposure to M. leprae. The Golden Jubilee issue of Leprosy in India is entirely devoted to these studies [1]. The patients respond normally to Cholera or Typhoid vaccines [2]. Their immune deficit is primarily their inability to react against $M$. leprae. Their lymphocytes do not undergo blast transformation in presence of M. Leprae and do not generate cytokines influencing macrophages to permit or not permit the multiplication of ingested M. leprae.

Leprosy patients manifest a spectrum. The multi-bacillary lepromatous leprosy (LL) patients have extreme immune deficit and are loaded with $M$. leprae, whereas the tuberculoid leprosy (TT) patients have limited lesions with hardly any bacilli. (Table 1) describes an experiment which we performed employing lymphocytes isolated from lepromatous (LL) patients or from tuberculoid (TT) leprosy patients. M. leprae requires host cells, which are the macrophages to multiply. Macrophages were developed from glass adhering monocytes in peripheral blood. It hardly mattered whether these originated from LL, TT patients or normal human volunteers. In order to assess the permissive multiplication of $M$. leprae, we took advantage of the fact that macrophages do not multiply in vitro, and do not synthesize 


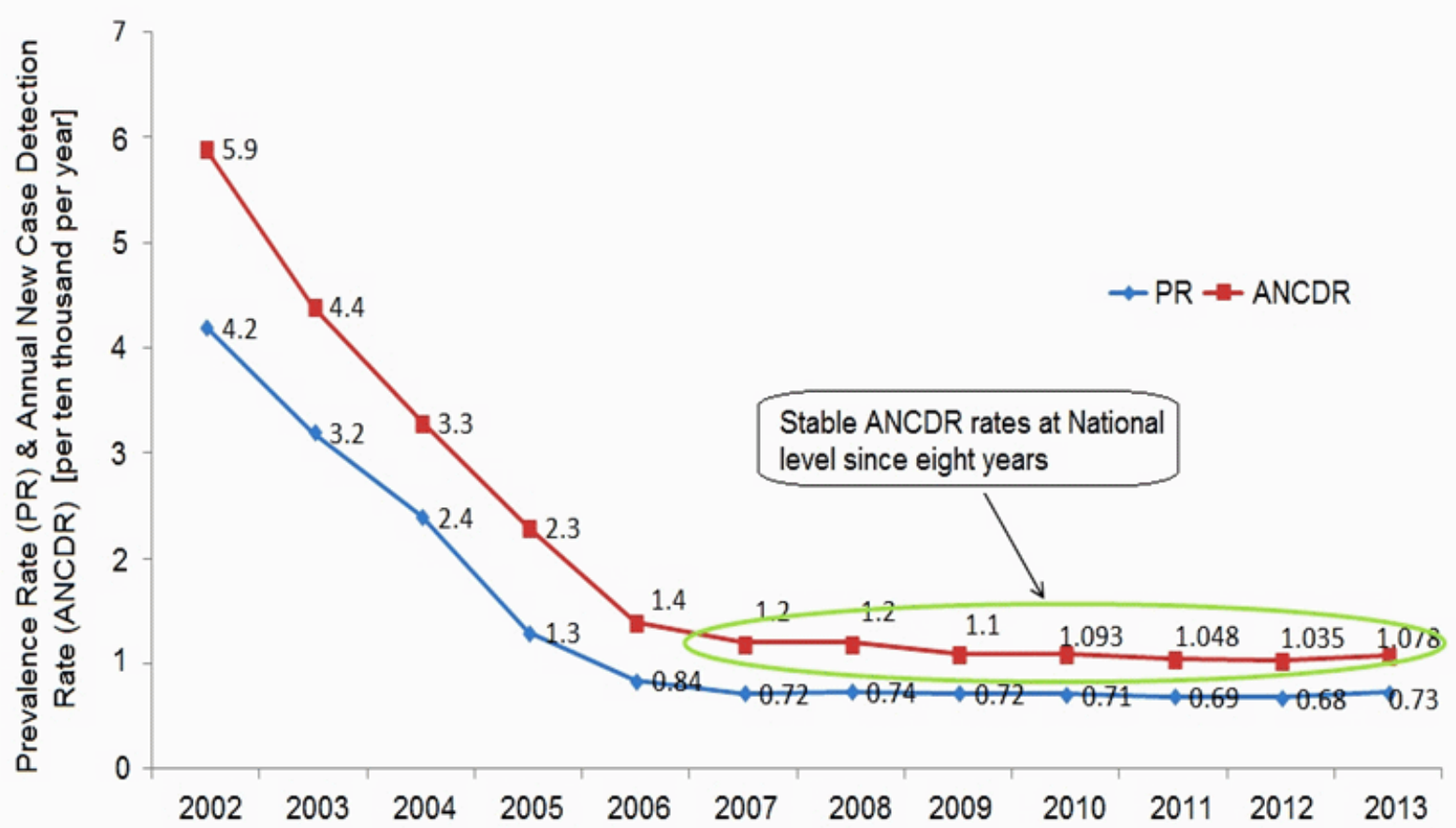

Figure 1: National Leprosy Eradication Programme (NLEP) data on Prevalence and Annual New Case Detection Rates in India.

Table 1: Mycobacterial multiplication in cultivated macrophages derived from peripheral blood monocytes of Leprosy patients (3)

\begin{tabular}{|c|c|c|c|}
\hline \multirow{2}{*}{ Patient No. } & \multirow{2}{*}{ Clinical Status } & \multicolumn{2}{|c|}{ CPM $^{3}$ H-thymidine incorporated per 5 $\mathbf{1 0}^{\mathbf{5}}$ phagocytic cells } \\
\cline { 3 - 4 } & Macrophages + Lymphocytes + M. leprae & Macrophages + M. leprae \\
\hline 1 & LL & 36,458 & 45,628 \\
\hline 2 & LL & 53,929 & 59,596 \\
\hline 3 & LL & 52,354 & 83,476 \\
\hline 4 & TT & 6,332 & 54,969 \\
\hline 6 & TT & 32 & 78,447 \\
\hline
\end{tabular}

DNA, whereas any microorganism, such as M. leprae must synthesize DNA for replication. Thus a pulse of ${ }^{3} \mathrm{H}-T h y m i d i n e$ could be employed to decipher the multiplication of M. leprae in a given situation. (Table 1) shows the important role of cytokines generated by 'competent' lymphocytes in response to M. leprae to limit or permit DNA synthesis as an index of permissive multiplication of $M$. leprae. While lymphocytes of LL patients do not restrict $M$. leprae to multiply, lymphocytes of TT patients restrict their multiplication.

For development of vaccine against leprosy, we adopted a heterologous approach, as the very nature of immune deficit is the inability of susceptible individuals to react to $M$. leprae. Thus using M. leprae as the basis of vaccine was illogical. We collected 16 strains of atypical mycobacteria, many were nonpathogenic and all of them were cultivable in vitro. Each one of them was investigated for its ability to cause blast transformation of lymphocytes from lepromatous leprosy patients. Five strains of mycobacteria, namely M.vaccae, M. phlei, M.gordonae, ICRC bacillus and an unidentified mycobacteria from atypical collection, which was codes by us Mw, were shortlisted for possessing the requisite capability [4].

Lepromin like preparations were made from these mycobacteria and evaluated for eliciting Delayed Hypersensitivity skin responses in both tuberculoid (TT) and lepromatous leprosy (LL) patients [5]. On basis of its ability to evoke not only positive response in TT but also in LL patients, Mw was chosen from these 5 strains for further investigations. Can it convert a lepromin negative LL patient who would continue to be lepromin negative to lepromin positivity status? Dr. Choudhary at the School of Tropical Medicine, Kolkata, conducted investigations 
in 32 individuals who were initially patients suffering from lepromatous leprosy and who were rendered bacterial negative after prolonged treatment with drugs. All of them continued to be lepromin negative, as is usually the case with LL patients. Drugs kill bacteria, but do not improve the immune status of the patient. After immunization of these patients with autoclaved Mw vaccine, surprisingly 20 of them became lepromin positive and what was more, this trait of positivity was retained on retest after several months. [5]

\section{Properties of MIP}

MIP as adjunct to the standard Multidrugs regime accelerates bacterial clearance and shortens the recovery period. [6] What is further impressive is that it clears granulomas. Patients recover into almost normal beings without the usual blemishes of the disease. (Figure. 2) shows a few patients who were treated with MIP along with the drugs.

MIP clears also the bacilli resident in peripheral nerves restoring normal sensitivity.

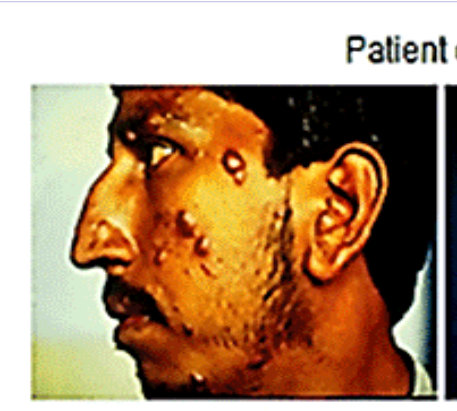

Initial

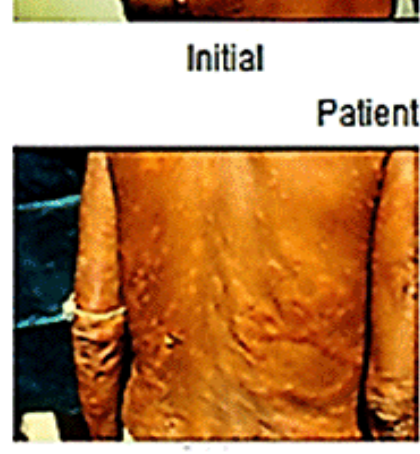

Initial

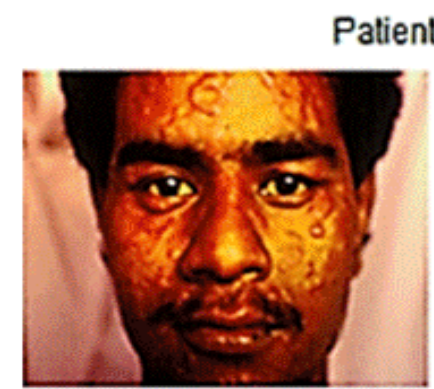

Initial

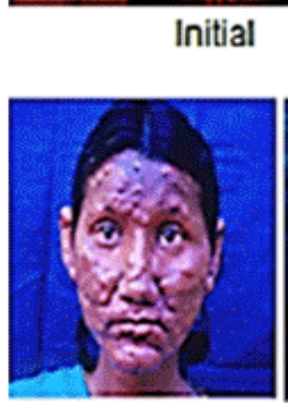

Initial

\section{Patient code: MD}

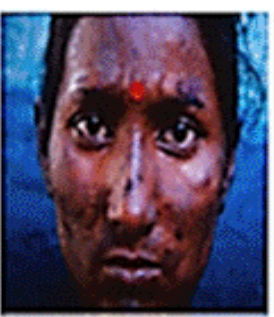

After 4 doses

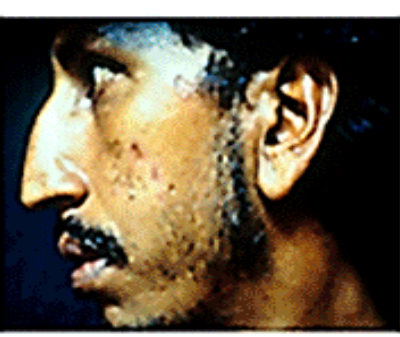

After 5 doses

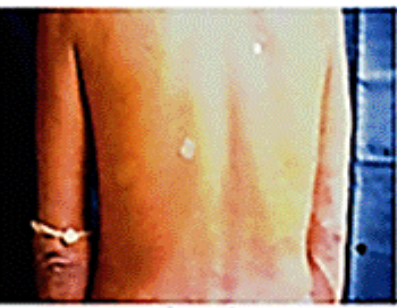

After 2 doses

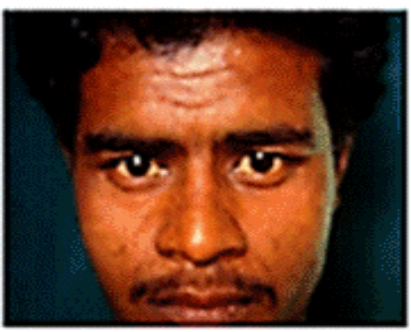

After 2 doses

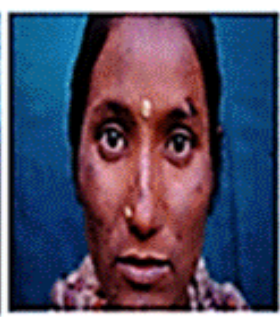

1 year thereafter

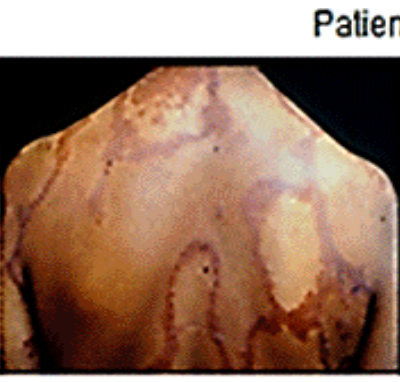

Initial

\section{Patient code: BWS}

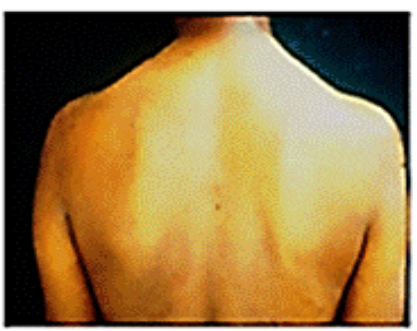

After 4 doses

Patient code: $\mathrm{CHB}$

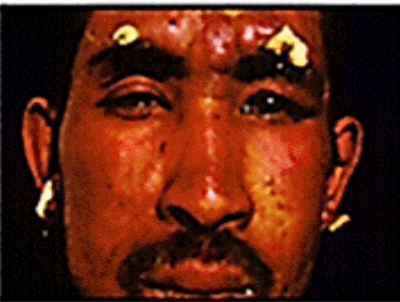

Initial

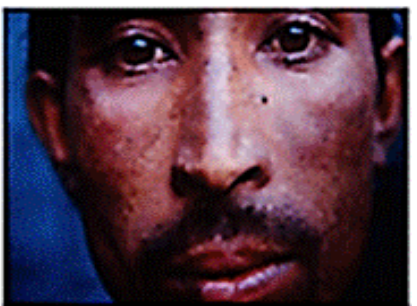

After 4 doses

Patient code: RAB

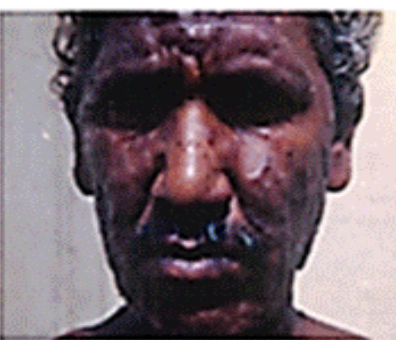

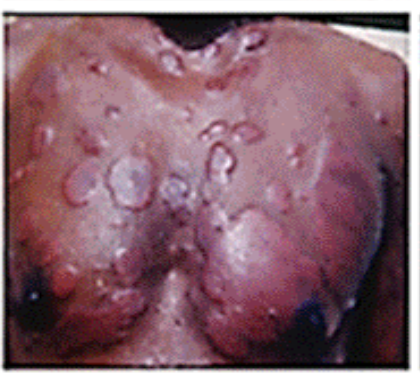

Initial
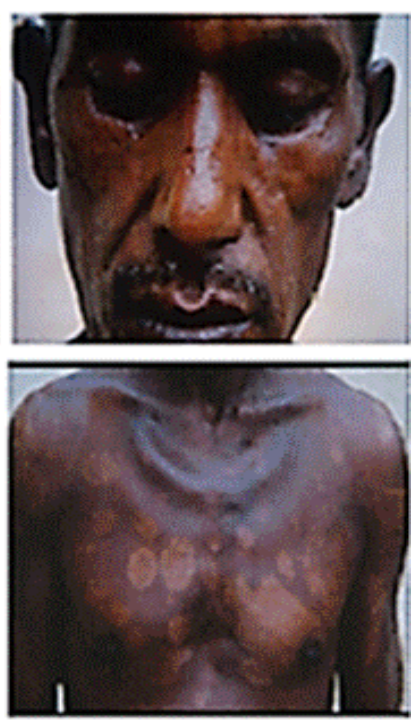

After 4 doses

Figure 2: Some representative cases of LL/BL multibacillary patients treated with MDT plus MIP (Mycobacterium indicus pranii) (6). 


\section{Additional Properties of MIP}

\section{i. Tuberculosis}

MIP shares antigens with not only M. leprae but also M. tuberculosis. It has been employed successfully in treatment of Category II "Difficult to treat" tuberculosis patients. [7]

\section{ii. Potent invigorator of both cellular and humoral immune response}

MIP being used as adjuvant in our Birth Control Vaccine against hCG, where its inclusion enhances the antibody titres substantially. [8]

\section{iii. Clearance of ugly ano-genital warts and lesions elsewhere on the body}

Given intralesionally, MIP clears dramatically ano-genital warts [9] and also lesions elsewhere such as feet. (Figure. 3) [10]

\section{iv. Preventive and therapeutic action on SP2/0 Myelomas and other cancers}

Prof. Dipankar Nandi at Indian Institute of Sciences, Bangalore, has reported both preventive and therapeutic action of MIP on SP2/0 myelomas in mice. [11]

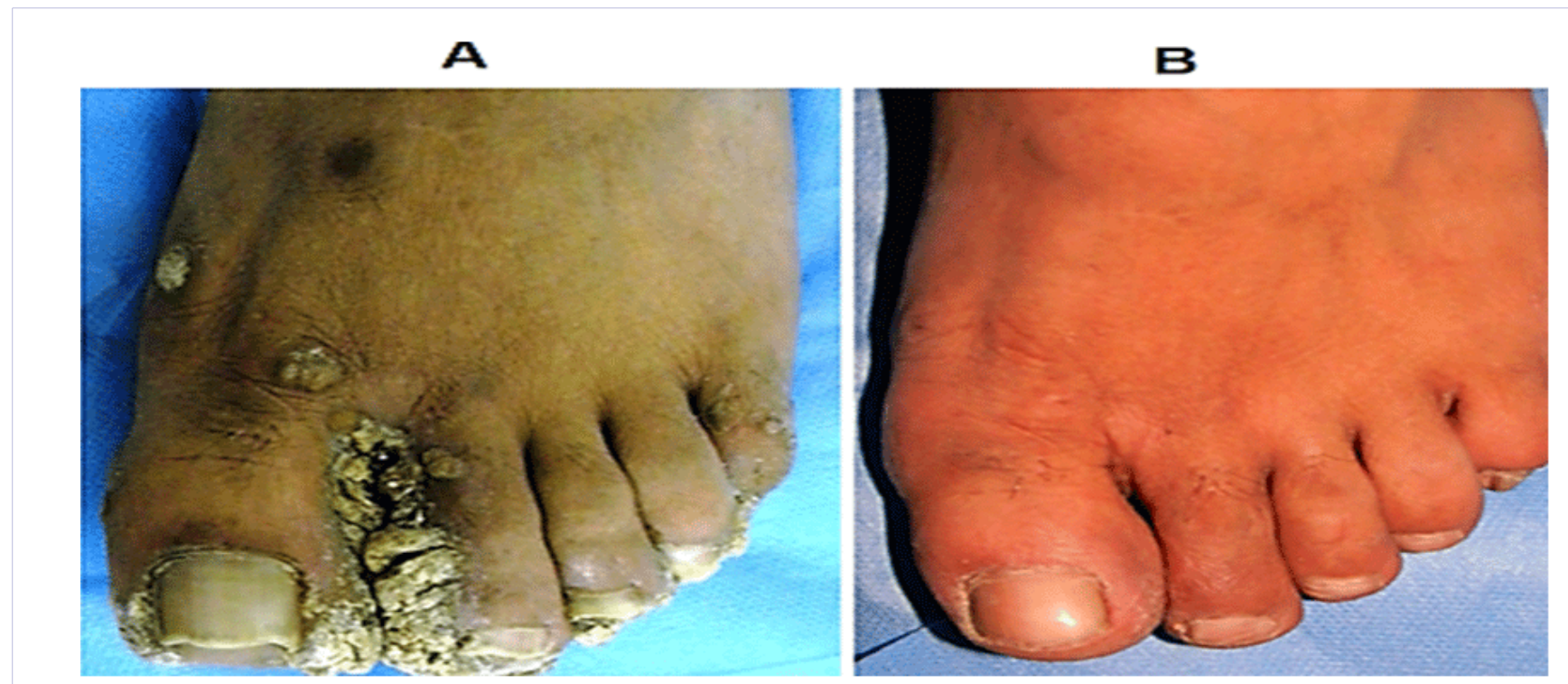

Figure 3: Cure by MIP of warts on feet. (A) Before treatment and (B) After 5 months of treatment with MIP (10).

Being an approved product by the Drugs Controller General of India (DCGI), and marketed by a Company to whom technology has been transferred, it is being used by patients suffering from various types of cancers.

\section{Genome sequence of MW (now named MIP)}

A network of 3 laboratories in India headed by Prof. SE Hasnain, Prof. Anil Tyagi and Prof. Akhilesh Tyagi have determined the genome sequence of Mycobacterium w [12,13]. Being a hitherto undescribed microorganism in International Collection, it has been named as Mycobacterium indicus pranii. Pran is my familiar name and nii is the National Institute of Immunology, New Delhi, of which I was the Founder Director, where much of the work on MIP was done under my guidance.

\section{Summary}

Described briefly in this communication is the background leading to the development of an immuno- therapeutic cum immuno-prophylactic vaccine against leprosy. The vaccine is an autoclaved suspension of non-pathogenic mycobacteria, whose genome sequence is now known and has been named as Mycobacterium indicus pranii (MIP). Along with drugs, it accelerates bacterial clearance and shortens recovery period of leprosy patients. It converts nearly $98 \%$ of normal lepromin negative healthy contacts to lepromin positivity status. On May 7, 2017, the Government of India has launched a field programme to eradicate leprosy in leprosy endemic districts where the patients will be immunized with the vaccine besides the usual drugs. Family members and contacts of the patients will receive 2 doses of the vaccine at 6 months interval.

Besides leprosy, MIP has been employed beneficially for treatment of Category II "Difficult to treat" tuberculosis patients. MIP given intralesionally clears ugly warts on various parts of the body. MIP is a potent invigorator of immune response and is being used as adjuvant in a vaccine against hCG for preventing unwanted pregnancies. MIP has also demonstrated preventive and therapeutic action against some cancers. MIP is approved by the DCGI and is being made available to public by a company to which it is licensed. 


\section{References}

1. Talwar GP, Mustafa AS, et al. 14 Papers in Golden Jubilee Issue Leprosy in India. 1978; 50:492-597

2. Talwar GP, Krishnan AD, Mehra VL, Blum EA, Pearson JM. Evaluation of cell mediated immune responses in untreated cases of leprosy. Clin Exp Immunol. 1972;12(2):195-203

3. Talwar GP, Krishnan AD, Jha P, Mehra V. Intracellular growth of an obligatory parasite Mycobacterium leprae: host bacterial interactions. Biochimie. 1974;56(2):231-237

4. Mustafa AS, Talwar GP. Five cultivable mycobacterial strains giving blast transformation and leukocyte migration inhibition of leukocytes analogous to Mycobacterium leprae. Lepr India. 1978;50(4):498-508

5. Chaudhuri S, Fotedar A, Talwar GP. Lepromin conversion in repeatedly lepromin negative BL/LL patients after immunization with autoclaved Mycobacterium w. Int J Lepr Other Mycobact Dis. 1983;51(2):159-68

6. Talwar GP. Leprosy is in principle eradicable: a possible approach. Curr Sci. 2014;106(10):1344-1345

7. Sharma SK, Katoch K, Rohit S, Balambal R, Nirmal Kumar J, Naresh patel, et al. Efficacy and Safety of Mycobacterium indicus pranii as an adjunct therapy in Category II pulmonary tuberculosis in a randomized trial. J Sci Rep. 2017;7: 3354. doi: 10.1038/s41598-017-03514-1
8. Purswani S, Talwar GP. Development of a highly immunogenic recombinant candidate vaccine against human chorionic gonadotropin. Vaccine. 2011;29912):2341-2348. doi: 10.1016/j. vaccine.2010.11.069

9. Gupta S, Malhotra AK, Verma KK, Sharma VK. Intralesional immunotherapy with killed Mycobacterium $w$ vaccine for the treatment of anogenital warts: an open-label pilot study. J Eur Acad Dermatol Venereol. 2008;22(9):1089-1093. doi: 10.1111/j.14683083.2008.02719.x

10. Singh S, Chouhan K, Gupta S. Intralesional immunotherapy with killed Mycobacterium indicus pranii vaccine for the treatment of extensive cutaneous warts. Indian. J Cutan Aesthet Surg. 2014;80(6):509-514. doi: 10.4103/0378-6323.144145

11. Rakshit S, Ponnusamy M, Papanna S, Saha B, Ahmed A, Nandi D. Immunotherapeutic efficacy of Mycobacterium indicus pranii in eliciting anti-tumor T cell responses: critical roles of IFNy. Int J Cancer. 2012;130(4):865-875. doi: 10.1002/ijc.26099

12. Talwar GP, Ahmed N, Saini V. The use of the name Mycobacterium w for the leprosy immunotherapeutic bacillus creates confusion with M. tuberculosis-W (Beijing strain): a suggestion. Infect Genet Evol. 2008;8(1):100-101. doi: 10.1016/j.meegid.2007.07.009

13. Saini V, Raghuvanshi S, Talwar GP, Ahmed N, Khurana JP, Hasnain SE, et al. Polyphasic taxonomic analysis establishes Mycobacterium indicus pranii as a distinct species. PLoS One. 2009;4:e6263. doi: 10.1371/ journal.pone.0006263 